\title{
Wogonin improves functional neuroprotection for acute cerebral ischemia in rats by promoting angiogenesis via TGF- $\beta 1$
}

\author{
Zhaohong Kong ${ }^{1,2}$, Qinglin Shen ${ }^{3}$, Jian Jiang ${ }^{2}$, Min Deng $^{2}$, Zhaohui Zhang $^{2}$, Gaohua Wang ${ }^{1}$ \\ ${ }^{1}$ Institute of Neuropsychiatry \& Mental Health Center, Renmin Hospital of Wuhan University, Wuhan 430061, China; ${ }^{2}$ Department of Neurology, \\ Renmin Hospital of Wuhan University, Wuhan 430060, China; ${ }^{3}$ Cancer Center, Renmin Hospital of Wuhan University, Wuhan 430060, China \\ Correspondence to: Zhaohui Zhang, MD, PhD. Department of Neurology, Renmin Hospital of Wuhan University, Wuhan 430060, China. \\ Email: zhzhqing1990@163.com; Gaohua Wang, MD, PhD. Institute of Neuropsychiatry \& Mental Health Center, Renmin Hospital of Wuhan \\ University, Wuhan 430060, China. Email: wgh6402@163.com.
}

\begin{abstract}
Background: Previous studies showed that wogonin is a potential candidate for more effective treatment of neuronal and inflammatory disease and could offer neuroprotective activity in various models, but all these studies were in vitro. Our research aimed to investigate the neuroprotective effect of wogonin on focal cerebral ischemia in rats and uncover its potential mechanism.

Methods: A total of 80 male SD rats were randomly divided into a sham operation group (Sham group, 20 rats), a normal saline group (NS group, 20 rats), and a wogonin intervention group (W2W group, 20 rats), while the remaining 20 rats were kept as a substitute. The model of focal cerebral ischemia (MCAO) was established by thread embolization. The neurological deficits were evaluated by the modified neurological deficit scale (mNSS). The laser confocal technique was used to observe the diameter, density, and total area of microvessel. Lastly, the expression of transforming growth factor- $\beta 1$ (TGF- $\beta 1$ ) was detected by Western blot.

Results: The mNSS scores of the NS group and $W n 2 W$ group were $6.57 \pm 1.13$ and $4.39 \pm 0.92$ respectively, and the difference between NS group and $\mathrm{Wn} 2 \mathrm{~W}$ group was statistically significant $(\mathrm{P}<0.05)$; the vascular diameter of the $W n 2 W$ group, Sham group, and NS group were $2.93 \pm 0.19,4.24 \pm 0.16$, and $3.56 \pm 0.22 \mu \mathrm{m}$ respectively, and the differences among these groups were statistically significant $(\mathrm{F}=102.142, \mathrm{P}<0.01)$. Furthermore, the differences in the vascular density $(\mathrm{F}=290.49, \mathrm{P}<0.01)$ and total microvessel area $(\mathrm{F}=163.08, \mathrm{P}<0.01)$ among these groups were also statistically significant. The expression of TGF- $\beta 1$ in ischemic brain tissue of the Sham group, NS group, and Wn2W group were $0.46 \pm 0.14,0.62 \pm 0.18$, and $0.94 \pm 0.21$ respectively, and the differences among these groups were statistically significant $(\mathrm{F}=102.142$, $\mathrm{P}<0.01)$.
\end{abstract}

Conclusions: Wogonin can markedly reduce nerve injury and improve nerve function in rats with cerebral ischemia, which may be related to the TGF- $\beta 1$ pathway.

Keywords: Wogonin; acute cerebral ischemia; neuroprotection; transforming growth factor- $\beta 1$ (TGF- $\beta 1$ )

Submitted Jul 16, 2019. Accepted for publication Oct 12, 2019.

doi: $10.21037 /$ atm.2019.10.70

View this article at: http://dx.doi.org/10.21037/atm.2019.10.70

\section{Introduction}

The cerebrovascular disease has become the most fatal among the three major death-related diseases that seriously endanger people's health, and ischemic cerebral vascular disease (ICD) accounts for about $80 \%$ of the nearly
2 million new cerebrovascular diseases (1). ICD brings a heavy burden to society and patients' families because of its high morbidity, high mortality, high disability rate, and inducement of depression. At present, acute intravenous thrombolysis and anti-platelet aggregation therapy are mainly used in clinic, and the other therapies, such as 
ultra-early reflow treatment and neuroprotection, have little clinical effect. Unexplained complex mechanisms of cerebral ischemic injury and lack of effective interventions are the main reasons for the lack of progress in ICD treatment.

Wogonin (5,7-dihydroxy-8-methoxyflavone), a flavonoid derived from the roots of Scutellaria Baicalensis Georgi, is an important bioactive component of flavonoids. Wogonin and its analogs have been widely used in Oriental Medicine for their anti-inflammatory antioxidant, and anti-anxiety effects, as well as their ability to alleviate stroke and provide neuroprotective effects. Wogonin can offer these wide ranges of effects because of its unique scavenging oxygenfree radicals and antioxidant activity (2-6). Wogonin is conducive to the dynamic maintenance and physiological reconstruction of blood vessels, which opens up a new way to enhance collateral circulation and vascular regeneration in ischemic diseases. Furthermore, wogonin is a potential plant for the effective prevention and treatment of atherosclerosis by regulating the function of vascular endothelial cells and vascular smooth muscle cells. In addition, various wogonin derivatives were examined as potential candidates for more effective treatment against neuronal and inflammatory disease (7) as well as neuroprotective activities in various models (8). Meanwhile, other studies have shown that wogonin has no or only minor neuroprotective effects on mature neurons $(7,9)$. However, all the studies concerned were in vitro in nature. Therefore, in the present study, we aimed to be the first to verify the neuroprotective effect of scutellarin in a live animal model. One previous study did find that TGF- $\beta 1$ could induce angiogenesis in rats after cerebral ischemic injury (10). However, the research on the effect and mechanism of wogonin on acute cerebral ischemia is rare.

In this study, we chose acute cerebral ischemia rat models and intervened with wogonin. We assessed the recovery of neurological function in each group after modeling 2 weeks by detecting the angiogenesis in the ischemic brain tissue using a laser confocal technique. We explored the possible molecular biological mechanism of wogonin's effect on acute cerebral ischemia by detecting the expression of TGF- $\beta 1$ in the ischemic brain tissue.

\section{Methods}

\section{Experimental animals}

A total of 80 male Sprague-Dawley (SD) rats [license number: SCXK (E) 2008-0004], SPF grade, aged 8-12 weeks, weighing 180-220 g, were purchased from the Animal Experimental Center of Wuhan University. The animals were caged in the experimental center of Zhongnan Hospital of Wuhan University at 40-60\% relative humidity and a temperature between $16-26^{\circ} \mathrm{C}$. The rats were fed by common fodder, and ate and drank freely in daylight. The rats' diet and drinking water were observed dynamically. The cage padding was replaced once every day. All the experimental procedures follow the guidance of the Ministry of Science and Technology on experimental animals.

All the rats were randomly divided into four groups according to their body mass: the sham group (20 rats), control group (20 rats), the $\mathrm{Wn} 2 \mathrm{~W}$ group (20 rats in the wogonin group), and the remaining 20 rats as substitutes.

\section{Preparation and detection of rat models}

The middle cerebral artery occlusion (MCAO) model was established by modified Longa-Zea thread embolization method (11). The specific operation process was done according to our previous research (12). To be considered a qualified model there needed to be the following manifestations: Horner's syndrome, characterized by small fissures and enophthalmos; and hemiplegia, characterized by the opposite forelimb. After successful modeling, the drug was intervened at the corresponding time point.

\section{Neurological deficit score}

The neuroprotective effect of wogonin was evaluated by the modified neurological severity score (mNSS). The scale can be used to quantify a variety of neurological deficits in experimental animals, including consciousness, olfaction, hearing, respiration, trigeminal nerve, corneal reflex, balance ability, limb muscle strength, environmental response, etc. (13).

As it relates to the improved neurological deficit scoring method (13), we performed the experiment one day before operation and 14 days after operation. The neurological function of the sham group, control group, and Wn2W group (10 rats in each group) were assessed by mNSS from motor, sensory, reflex, and balance tests, and neurological deficits ranged from normal to severe with a score from 0 to 18. Each missing item in the reflex test was assessed with a score of 1 and more than 13 as severe injuries. The higher the NSS score, the greater the degree of neurological 
deficits. Six rats that died of cerebral hemorrhage and severe neurological impairment caused by operation errors were excluded. The rats with an mNSS score of 3-14 were selected as candidaters for random substitution in the later experiments.

\section{Drug intervention}

The suspension of wogonin was prepared by the dissolution of wogonin by $0.9 \%$ sodium chloride solution $(20 \mathrm{mg} / 100 \mathrm{~mL})$. Rats in the control group were given the same volume control (10 mL/kg, each rat was converted to about $2 \mathrm{~mL} / \mathrm{d})$ 2 hours after the model was made, and the rats in the sham group were given no drugs, only routine feeding, and observation.

Wogonin was given orally once a day (50 micro mols/L, $10 \mathrm{~mL} / \mathrm{kg} /$ day), and NS was used as the negative control group (given the same volume of NS solution) once every 24 hours for 14 consecutive days.

\section{Confocal three-dimensional brain angiography}

Six rats in each group were anesthetized 2 weeks after modeling (12). The plasma was labeled with FITC-low molecular weight dextran (relative molecular weight $2 \times 10^{6}$, $50 \mathrm{mg} / \mathrm{mL} / \mathrm{kg}$, Sigma Company) through the femoral vein. The brain tissues were quickly cut out, and the brain tissues were fixed in an icebox with $4 \%$ paraformaldehyde for 24-48 $\mathrm{h}$ and placed out of the light. The specimens were coronally sliced with a vibrating microtome. The optic chiasma was selected and sliced continuously to the cerebral end. The thickness of the slice was $100 \mu \mathrm{m}$. One hundred layers were scanned by laser scanning confocal microscope. The volume of green fluorescence indicated the amount of plasma perfusion. The images obtained under $40 \times$ microscope were used to analyze the vessels with a diameter of less than $8 \mathrm{~mm}$. The morphology, inner diameter, density, and a number of fluorescent substances per unit area were observed.

\section{Apoptosis analysis}

The transplanted cells and their intracranial proliferation were detected after an intervention of 2 weeks ( $\mathrm{n}=6$ /group). The specific operation process was performed according to our previous research (12).

TUNEL assay was used to detect the apoptosis of cells in the Sham group, the NS group, and the $\mathrm{Wn} 2 \mathrm{~W}$ group.

\section{Observation under a transmission electron microscope}

The ischemic brain tissues were harvested 2 weeks after intervention. Firstly, the brain tissues were harvested from the ischemic area, fixed with $2.5 \%$ glutaraldehyde, rinsed with $0.1 \mathrm{M}$ phosphoric acid buffer three times, rinsed with osmium acid (O3O 4.1\%) and fixed with $0.1 \mathrm{M}$ phosphoric acid buffer three times, dehydrated with graded alcohol, and then embedded with epoxy resin two times $(15 \mathrm{~min})$, soaked in EPON812 and acetone mixtures (v:v 1:1) for $30 \mathrm{~min}$, pure-embedding-liquid soaked for $1 \mathrm{~h}$ and pureembedding-fluid solidified at $37^{\circ} \mathrm{C}$ for $24 \mathrm{~h}$, and at $60^{\circ} \mathrm{C}$ for $48 \mathrm{~h}$. The LKB-V ultra-thin section machine, produced by Sweden BROMMA Company, was stained with uranium and lead (uranium peroxide acetate, lead citrate). Ten visual fields were randomly selected and observed under a transmission electron microscope ( $\mathrm{n}=6$ /group).

\section{Immunobistochemical (IHC) staining detected the expression of TGF- 1 in ischemic brain tissue}

IHC staining was performed on 5 rats in each group 2 weeks after modeling. The procedure of tissue perfusion and fixation was as follows. Firstly, the rats were anesthetized by intraperitoneal injection of $10 \%$ chloral hydrate $(300 \mathrm{mg} / \mathrm{kg})$. After continuous perfusion of paraformaldehyde for $30 \mathrm{~min}$, the brain tissues were taken from the rats and fixed with $4 \%$ paraformaldehyde, dehydrated, and embedded in paraffin. TGF- $\beta 1$ IHC was performed. The first antibody was antiTGF- $\beta 1,1: 200$, the slices were dewaxed to water, incubated, dried, and mixed with an anti-EGF antibody overnight at $4{ }^{\circ} \mathrm{C}$ and incubated at $37{ }^{\circ} \mathrm{C}$. Take $1 \mathrm{~mL}$ distilled water, 1 drop of $\mathrm{A}, \mathrm{B}$, and $\mathrm{C}$ reagent in the kit, then mix it and drop it to the center of the slice, shake gently at last. IHC images were analyzed by Image-Pro Plus 6.0 software, and integrated optical density (IOD) was measured.

Each section of each group was randomly selected from the cortex, striatum, and hippocampal CA1 area of the same cortical ischemia area of three 400 -fold visual fields for photography. Image-Pro Plus 6.0 software was used to analyze the IHC images, and the IOD of each picture was obtained. The average value of all photos in each group represents the IOD of the group, expressed by mean \pm standard deviation.

\section{Western blot detected the expression of TGF- 1 in ischemic brain tissue.}

We chose 6 groups for two-time points, and each group 
Page 4 of 10

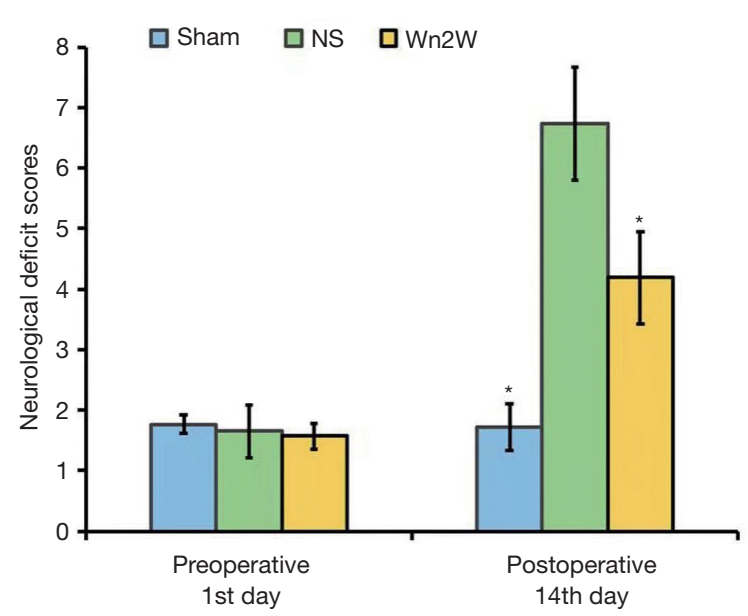

Figure 1 Neurological deficit score of rats among the Sham group, NS group, and $\mathrm{Wn} 2 \mathrm{~W}$ group at preoperative day 1 and postoperative day 14 . *, $\mathrm{P}<0.05 \mathrm{NS}$ group versus ham or $\mathrm{Wn} 2 \mathrm{~W}$ group.

(sham group, control group, and $\mathrm{Wn} 2 \mathrm{~W}$ group) took 5 rats 2 weeks after modeling for TGF- $\beta 1$ Western blot. We took frozen tissue, lysis, and homogenate from around ischemic areas. Sample buffers of equal volume $(0.125 \mathrm{~mol} / \mathrm{L}$ Tris$\mathrm{HCl} 4 \%$ SDS, $2 \%$ beta-mercaptoethanol, $20 \%$ glycerol, $0.2 \%$ bromophenol blue, PH 6.8 ) were added to the homogenate. Samples were boiled for $3 \mathrm{~min}$, centrifugated at $10,000 \mathrm{~g}$ for $10 \mathrm{~min}$ at $4{ }^{\circ} \mathrm{C}$ to obtain the supernatant. The protein concentration was determined by ultraviolet spectrophotometer, and the total amount of total protein containing $50 \mu \mathrm{g}$ was determined. A total of 15 SDS-PAGE samples was taken for SDS-PAGE electrophoresis (Bio-Bad) to complete the electrotransfer. The PVDF film was sealed at room temperature and then sealed with first and second antibodies. The substrate (1:2 mixture, Protein Detector LumiGLO Western blotting Kit) was added to the film ( $1 \mathrm{~min}$ ), and the film was exposed for $10 \mathrm{~min}$. The PVDF film was developed by elution solution $(62.5 \mathrm{mmol} / \mathrm{L}$ Tris-HCL, PH6.8, $20 \%$ SDS, $100 \mathrm{mmol} / \mathrm{L}$ betamercaptoethanol) in Ant mice. I-GAPDH monoclonal antibody $(1: 5,000)$ electrophoresis was used as an internal reference. The images were scanned into the computer and processed by image analysis software, and the corresponding optical density values were quantitatively analyzed.

\section{Statistical analysis}

The data of each group are expressed as mean \pm standard
Kong et al. Wogonin improves neuro-function via TGF- $\beta 1$ in stroke

deviation $(\bar{x} \pm \mathrm{S})$. The paired $t$-test was used for comparison between the two samples, and the one-way ANOVA and Kruskal-Wallis test (with post hoc tests for two-group comparisons if needed) was used for comparison among the groups. All statistical calculations were completed by SPSS21.0 software, and $\mathrm{P}<0.05$ was considered as statistical difference.

\section{Results}

\section{Neurological deficit score of rats}

One day before the model was made, the mNSS scores of the sham operation group, the control group, and the $\mathrm{Wn} 2 \mathrm{~W}$ group were $1.67 \pm 0.16,1.65 \pm 0.43$, and $1.57 \pm 0.21$. There was no significant difference between the two groups $(\mathrm{F}=0.82, \mathrm{P}>0.05)$, indicating that the brain nerves of the rats in each group were not damaged and the nerve function was normal. The experimental foundation was basically the same.

Two weeks after operation, the mNSS score of shamoperated rats had no significant change $(1.72 \pm 0.39)$. The mNSS scores of the control group, Wn2W group, and $\mathrm{Wn} 3 \mathrm{~W}$ decreased in varying degrees. The score of $\mathrm{Wn} 2 \mathrm{~W}$ groups $(4.19 \pm 0.76)$ was significantly lower than that of the control group $(6.77 \pm 1.03)$. There was a significant difference between the two groups $(\mathrm{P}=0.015)$, indicating that the recovery of nerve function in the $\mathrm{Wn} 2 \mathrm{~W}$ group was obvious: it was better than the control group (Figure 1).

\section{Three-dimensional confocal angiography}

Three-dimensional confocal analysis of rat brain tissue after 14 days showed that the vascular diameter of the Wn2W group, Sham group, and NS group was $2.93 \pm 0.19$, $4.24 \pm 0.16$, and $3.56 \pm 0.22 \mu \mathrm{m}$ respectively, and the differences among those group were statistically significant $(\mathrm{F}=102.142$, $\mathrm{P}<0.01$ ); the vascular density of the $\mathrm{Wn} 2 \mathrm{~W}$ group, Sham group, and NS group were $232.68 \pm 12.54$ pcs/0.002, $131.88 \pm 6.32 \mathrm{pcs} / 0.002$, and $176.26 \pm 10.87 \mathrm{pcs} / 0.002 \mathrm{~mm}^{3}$ respectively, and the differences among these groups were statistically significant $(\mathrm{F}=290.49, \mathrm{P}<0.01)$; the total microvessel area of the $\mathrm{Wn} 2 \mathrm{~W}$ group, Sham group, and NS group were $89,154 \pm 3,298 \mathrm{\mu m}^{2} / 0.002 \mathrm{~mm}^{3}, 64,923 \pm 2,804 \mathrm{\mu m}^{2} / 0.002 \mathrm{~mm}^{3}$, and $75,368 \pm 3,519 \mu^{2} / 0.002 \mathrm{~mm}^{3}$ respectively, and the differences among these groups were statistically significant $(\mathrm{F}=163.08, \mathrm{P}<0.01)$.

These results suggest that angiogenesis exists in the peri- 

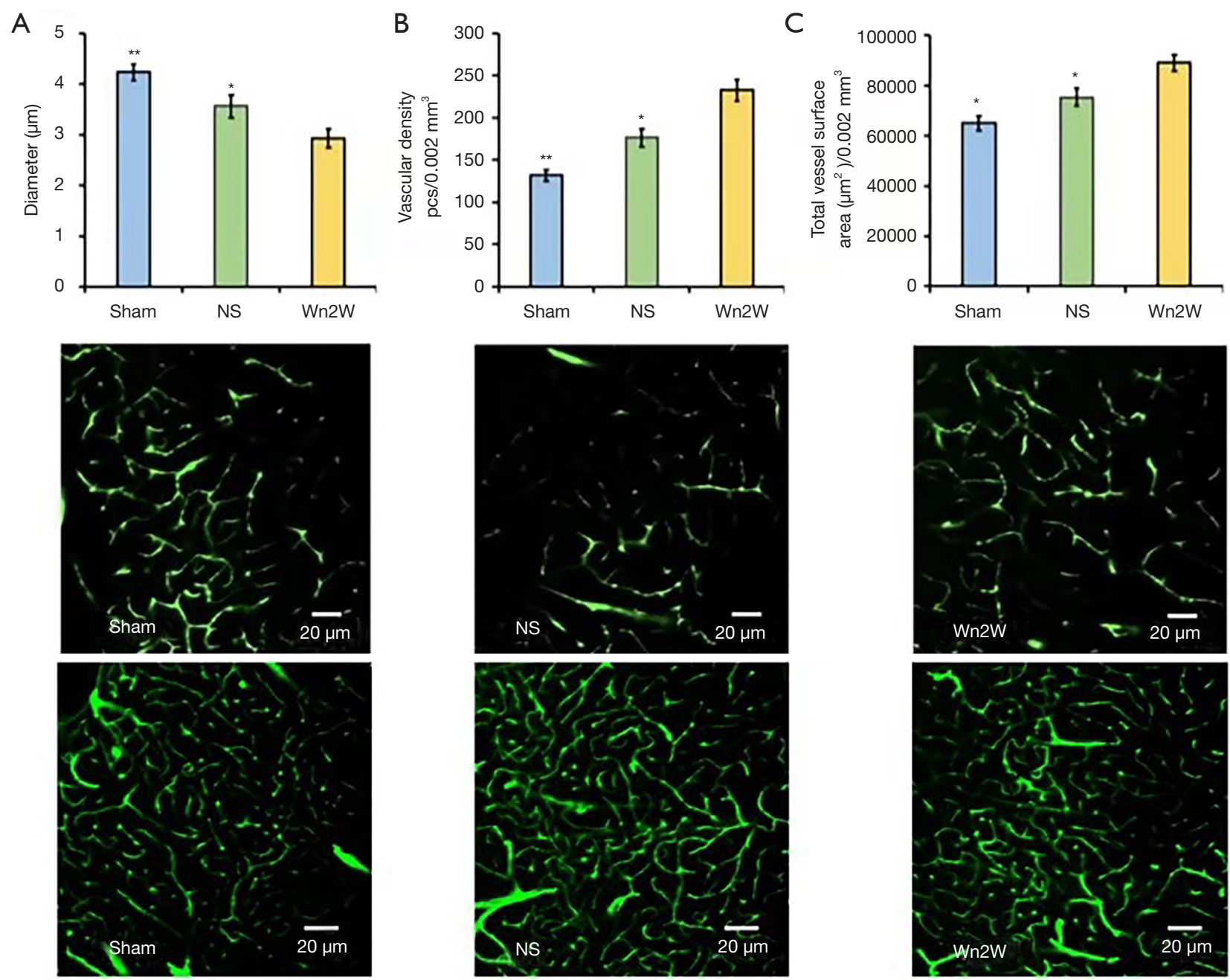

Figure 2 Three-dimensional confocal analysis of rat brain tissue after 14 days. (A) The differences of the vascular diameter of the Wn2W group, Sham group, and NS group; (B) the differences of the vascular density of the Wn2W group, Sham group, and NS group; (C) the differences of the total microvessel area of the Wn2W group, Sham group, and NS group. *, $\mathrm{P}<0.05 \mathrm{Wn} 2 \mathrm{~W}$ group versus Sham or NS group; **, $\mathrm{P}<0.01 \mathrm{Wn} 2 \mathrm{~W}$ group versus Sham or NS group.

ischemic area of the rat brain 14 days after cerebral ischemia and the drug intervention used in this study can clearly promote angiogenesis after cerebral ischemia (Figure 2).

\section{Apoptosis analysis}

TUNEL assay showed that green staining cells could be seen in all groups, and the morphology of cells was different, indicating that these cell lines were apoptotic cells. Statistical analysis showed that the number of apoptotic cells in the NS group was significantly higher than that in the Sham group and $\mathrm{Wn} 2 \mathrm{~W}$ group. It can be speculated that wogonin can significantly reduce the number of neuronal apoptosis in ischemic brain tissue, and has a neuroprotective effect (Figure 3).

\section{Cell observation under a transmission electron microscope}

After acute ischemia, the rats had deformed neurons and indistinguishable light and dark neurons, with deformed nuclei, irregular shape, increased heterochromatin, increased electron density, and unclear structure. The organelles in the cytoplasm were reduced or deformed, and the structure was difficult to discern. In the Sham group, the cell gap was clear, homogeneous, and had no effusion. In the NS group, the cell gap was widened, and a large amount of 


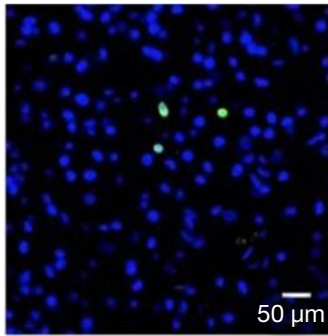

Sham

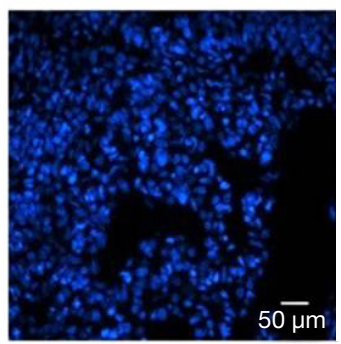

Sham

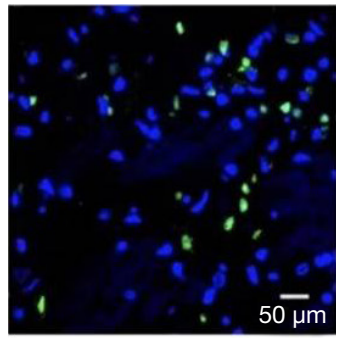

NS

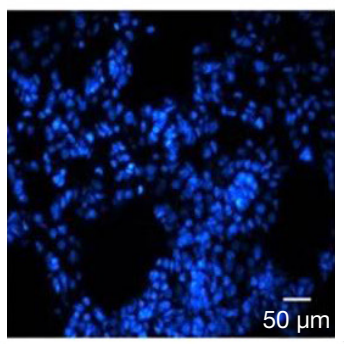

NS

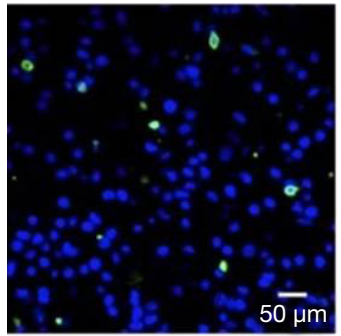

Wn2W

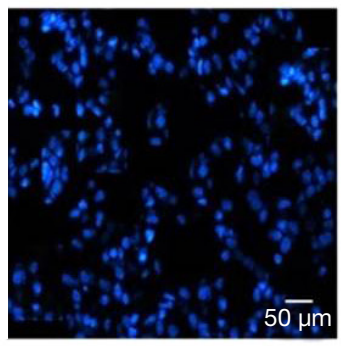

Wn2W

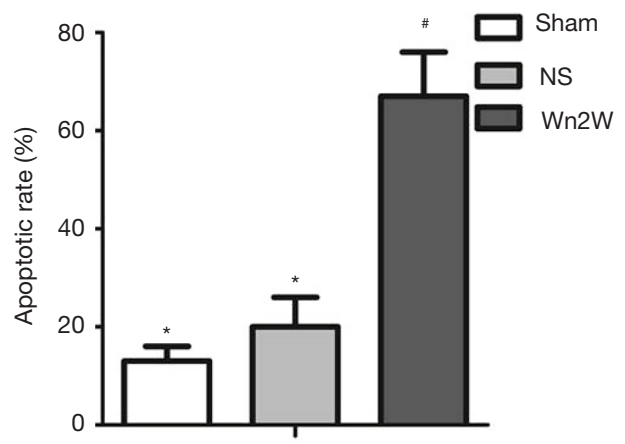

Figure 3 Apoptosis analysis among the Sham group, NS group, and Wn2W group. Statistical analysis showed that the number of apoptotic cells in the NS group was significantly higher than that in the Sham group and Wn2W group. *, $\mathrm{P}<0.05 \mathrm{Wn} 2 \mathrm{~W}$ group versus Sham or NS group; ${ }^{\#}, \mathrm{P}<0.01 \mathrm{Wn} 2 \mathrm{~W}$ group versus Sham or NS group.

edema fluid around it appeared as a low electron-density bright area, which is a typical manifestation of inflammation and edema of the nerve cells. In the $\mathrm{Wn} 2 \mathrm{~W}$ group, the width of the cell gap and the degree of effusion were markedly reduced (Figure 4).

\section{Detection of TGF-ß1 level in brain tissue}

IHC staining showed that TGF- $\beta 1$ positive cells had the morphological characteristics of neurons, mainly distributed in the cerebral cortex, showing dark brown-yellow cytoplasm, more around the ischemic area, but with few in the ischemic center, and expressed in neurons, glial cells, and vascular endothelial cells.

The expression of TGF- $\beta 1$ was observed two weeks after ischemia-reperfusion. The IOD of the $\mathrm{Wn} 2 \mathrm{~W}$ group was significantly higher than that of the Sham group and the NS group at 14 days after the operation $(\mathrm{F}=647.04, \mathrm{P}<0.01)$. It was further confirmed that the expression of TGF- $\beta 1$ was up-regulated after cerebral ischemia, and TGF- $\beta 1$ was upregulated after drug intervention. The level of expression increased significantly (Figure 5).

\section{Detection of the expression of TGF-ק1 in ischemic brain tissue.}

The expression of TGF- $\beta 1$ in ischemic brain tissue of the Sham group, NS group, and Wn2W group were
$0.46 \pm 0.14,0.62 \pm 0.18$, and $0.94 \pm 0.21$ respectively, and the differences among these groups were statistically significant $(\mathrm{F}=102.142, \mathrm{P}<0.01)$. The NS group and the $\mathrm{Wn} 2 \mathrm{~W}$ group were significantly higher than those of the Sham group $(\mathrm{P}=0.001)$. The $\mathrm{Wn} 2 \mathrm{~W}$ group was significantly higher than those of the NS group $(\mathrm{P}=0.006)$ (Figure $6 A$ ).

Western blot of TGF- $\beta 1$ of the ischemic brain tissue in the Sham group, NS group, and $\mathrm{Wn} 2 \mathrm{~W}$ group are shown in Figure $6 B$.

\section{Discussion}

To date, many researchers have focused on the mechanism of angiogenesis and its effective regulatory means after cerebral ischemia. They have strived to find new therapeutic drugs and interventions to promote angiogenesis for restoring neurological deficit function and improving the quality of life of patients after cerebral ischemia. Angiogenesis plays an important role in the process of neural protection and nerve repair in cerebral ischemia. In the early stage of cerebral ischemia, there is still an "ischemic penumbra" around the infarct. The damage of neurons in the ischemic penumbra can be reversed, and the damage of cerebral ischemia can be reduced to the lowest point if the blood supply is restored in time. Therefore, the recovery of nerve function after stroke is 


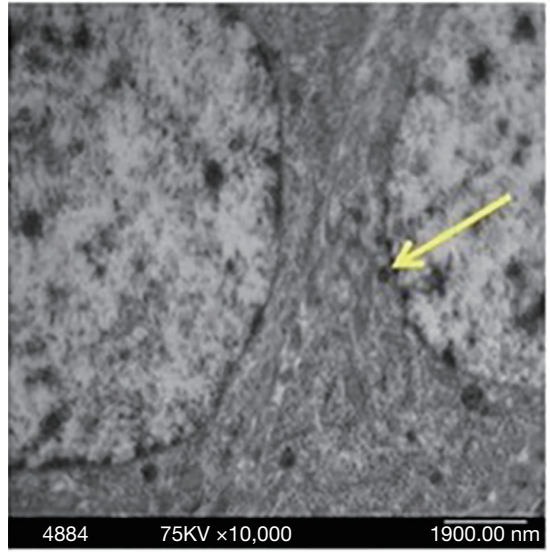

Sham

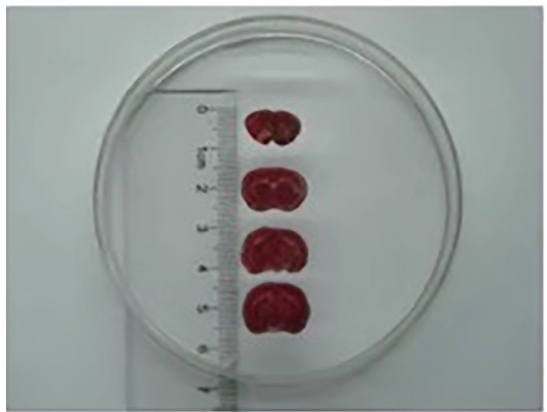

Sham

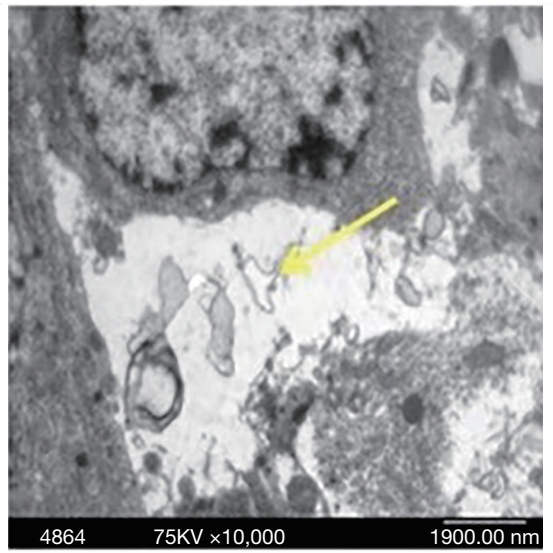

NS

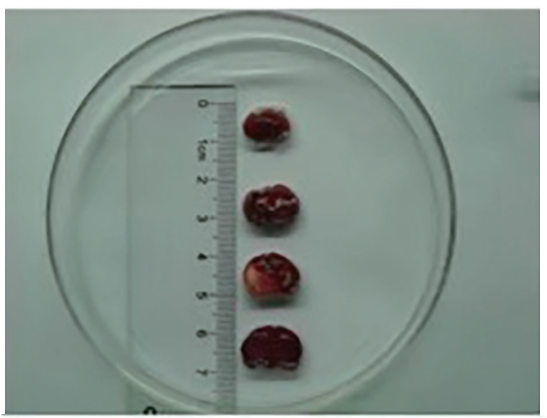

NS

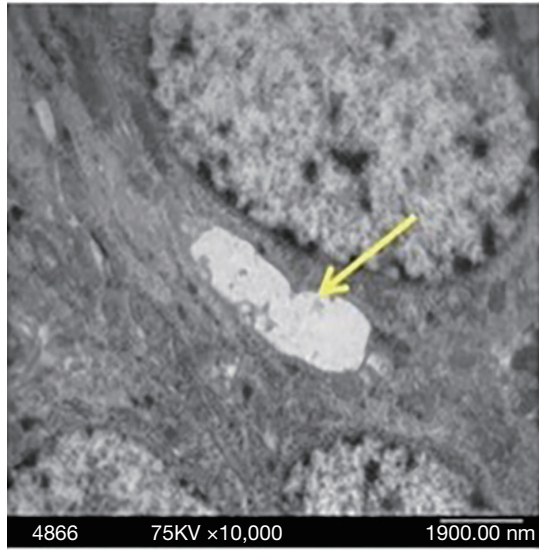

Wn2W

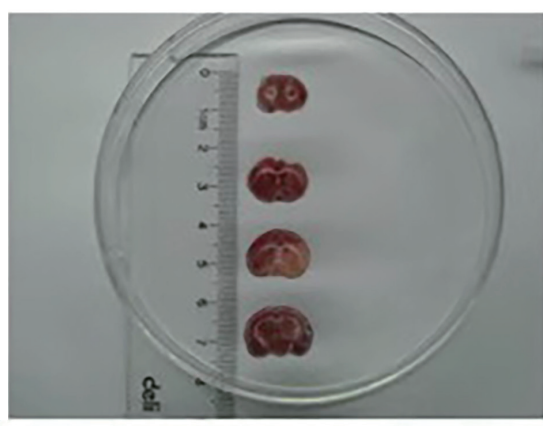

Wn2W

Figure 4 Cell observation under transmission electron microscope among the Sham group, NS group, and Wn2W group. The result showed that in the Sham group, the cell gap was clear, homogeneous, and had no effusion. In the NS group, the cell gap was widened, and a large amount of edema fluid around it appeared as a low electron-density bright area. However, in the Wn2W group, the width of the cell gap and the degree of effusion were obviously reduced.

the synergistic effect of neuroplasticity, vascular structure, and function reconstruction. Promoting angiogenesis and nerve remodeling is an important strategy to improve the recovery of nerve function after stroke.

To further explore the molecular biological mechanism of the neuroprotective effect of wogonin, we detected the expression levels of TGF- $\beta 1$ in each group. Our results showed that TGF- $\beta 1$ levels in the $W n 2 W$ group were significantly higher than those in the NS group and Sham group. Confocal microscopy showed that angiogenesis in the $\mathrm{Wn} 2 \mathrm{~W}$ group was significantly better than that in the NS group and the Sham group at 2 weeks. The cumulative optical density of TGF- $\beta 1$ positive cells and the gray value of Western blot protein bands in the $\mathrm{Wn} 2 \mathrm{~W}$ group were significantly higher than those in the Sham group and the NS group, which suggests that the intervention of wogonin after cerebral ischemia could promote the expression of TGF- $\beta 1$ significantly. The accumulated IOD and protein band gray value of TGF- $\beta 1$ positive cells in $\mathrm{Wn} 2 \mathrm{~W}$ group were significantly higher than those in the NS group, indicating that wogonin could significantly up-regulate the expression of TGF- $\beta 1$ protein after cerebral ischemia and that it was also directly related to the increase of vascular permeability of TGF- $\beta 1$. Our results showed that wogonin could alleviate the nerve injury and improve the nerve function in rats with cerebral ischemia-reperfusion injury, and had a neuroprotective effect, which may be related to TGF- $\beta 1$-mediated $s$ angiogenesis.

Wogonin could promote the angiogenesis of ischemic brain, and the effect of wogonin intervention was gradually effective over time, which may be related to the microenvironment, which can promote the migration and survival of vascular endothelial cells and the formation of new blood vessels in the ischemic region. We speculated that wogonin could improve neurological function by effectively alleviating ischemic neuronal damage, promoting 
Page 8 of 10

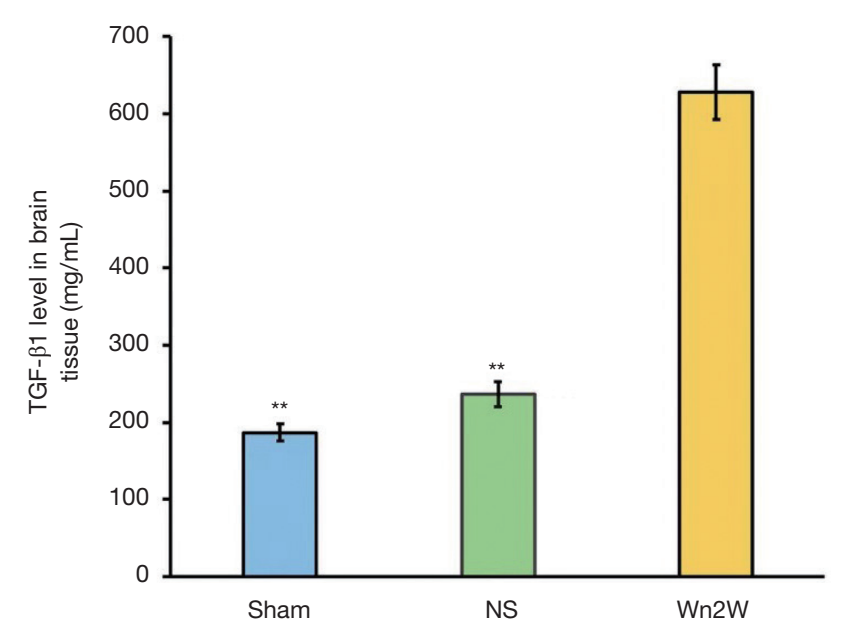

Figure 5 Detection of TGF- $\beta 1$ level in brain tissue among the Sham group, NS group, and Wn $2 \mathrm{~W}$ group. **, $\mathrm{P}<0.01 \mathrm{Wn} 2 \mathrm{~W}$ group versus the Sham or NS group.

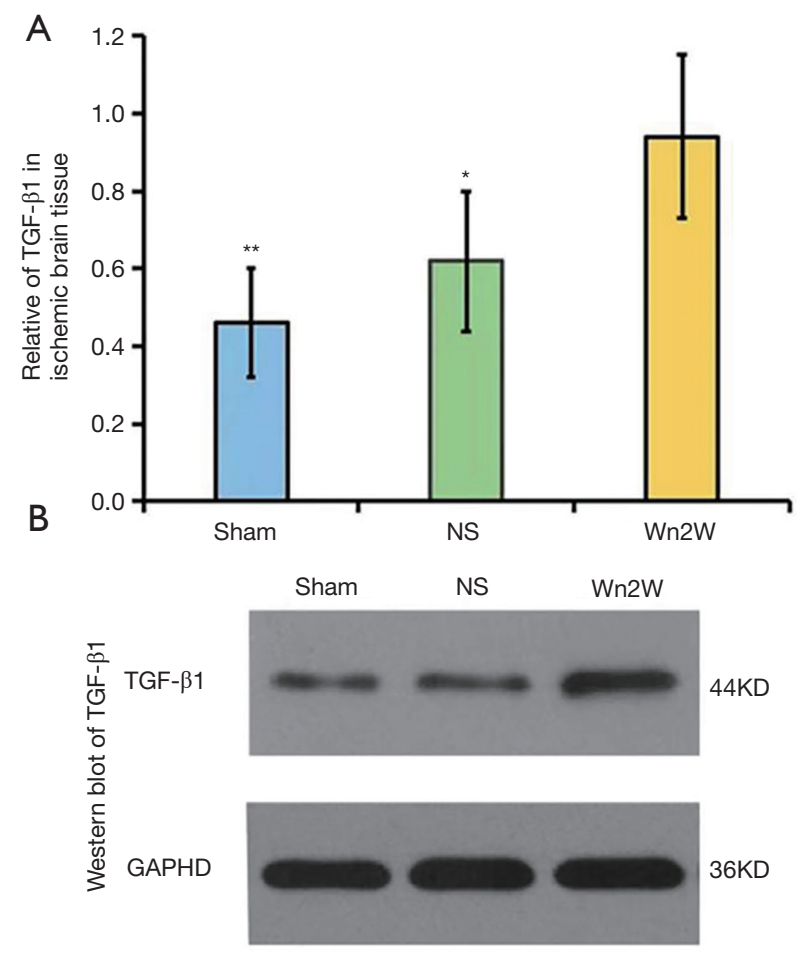

Figure 6 Detection of the expression of TGF- $\beta 1$ in ischemic brain tissue among the Sham group, NS group, and Wn2W group. (A) Relative expression of TGF- $\beta 1$ in ischemic brain tissue; (B) Western blot of TGF- $\beta 1$ in ischemic brain tissue. *, $\mathrm{P}<0.05$ $\mathrm{Wn} 2 \mathrm{~W}$ group versus the Sham or NS group; ${ }^{* *}, \mathrm{P}<0.01 \mathrm{Wn} 2 \mathrm{~W}$ group versus the Sham or NS group.
Kong et al. Wogonin improves neuro-function via TGF- $\beta 1$ in stroke

the formation of ischemia-induced neurovascular units, effectively secreting neurotrophic factors, and promoting angiogenesis and nerve repair in ischemic brain tissue.

We know that the specific mechanism of the wogonin improvement of neuroprotective function in rats is still unclear. We speculate that the possible reasons for this are as follows. Wogonin can play a neuroprotective role in ischemic neurons through a specific unknown receptor after entering the rat body. These receptors may be expressed in neurons, which further activate intracellular signal transduction pathways, and promote vascular regeneration and cell proliferation, and thus play a neuroprotective role. Previous studies have shown that wogonin may play a neuroprotective role mainly by inhibiting microglial overactivation $(5,14,15)$, and by inactivating the NF-B signaling pathway (5). Wogonin can improve the learning and memory ability of rats induced by amanithine, and can also increase the survival of cells and the activity of cholinergic neurons (16). In addition, some studies have found that wogonin induces the differentiation of neuroprotective components in vivo and in vitro, which is also involved in the protective effect of wogonin on neurons of damaged brain cells (17). In this study, we found that the proliferation of cells increased significantly in the $\mathrm{Wn} 2 \mathrm{~W}$ group, indicating that wogonin can effectively promote the regeneration of brain cells in the ischemic area, and thus plays a neuroprotective role. Unfortunately, there is no follow-up mechanism study in this experiment. Wogonin can play a neuroprotective role by inducing oxidative stress, lipid peroxidation, anti-inflammation, alleviating inflammatory reaction, and providing anti-immunity. These protective effects can effectively alleviate the injury of nerve cells and vascular endothelial cells, and then facilitate the recovery of local blood flow after ischemia, thereby reducing the damage of nerve function. Wogonin can induce and counteract the toxicity of cultured rat hippocampal cells in oxygen-glucose stripping environment, induce lipid peroxidation induced by nicotinamide adenine dinucleotide phosphate (NADPH), and oxidative stress induced by primary cultured rat cortical cells and human neuroblasts SH-SY5Y cells. In our experiments, transmission electron microscopy also showed that wogonin could effectively reduce the inflammatory edema of nerve cells, which is consistent with previous research results $(18,19)$. Wogonin can play a neuroprotective role by exerting an anti-apoptotic effect. Studies have shown that wogonin can inhibit 
the apoptosis of tumor cells and the death of nerve cells. Wogonin showed no toxicity or low toxicity to normal cells or tissues (20,21). Many studies have shown that wogonin has inhibitory effects on the growth of various tumor cell lines (22). Wang et al. reported that wogonin inhibited Raji cells in a dose and time-dependent method (23). Similar results were also found in other tumor cell lines, such as sarcoma S180 cell (24) and hepatoma cell SMMC-7721 (25). Our previous studies have shown that wogonin can effectively reduce the apoptosis of smooth muscle cells and play a protective role in atherosclerosis (10). In our study, we also found that wogonin can reduce apoptosis, but with no quantitative detection.

In addition, in our study, we found that wogonin plays a protective role by promoting the regeneration of blood vessels in the injured brain and forming neurovascular units. Our results showed that the total area of microvessels in the $\mathrm{Wn} 2 \mathrm{~W}$ group was significantly increased, and the effective blood flow was significantly restored. It has been reported that angiogenesis can promote the recovery of nerve function. The possible mechanisms may be as follows. Firstly, promoting and restoring the blood supply can reduce the ischemic area and delay the infarction of nerves in the ischemic area. Secondly, vascular endothelial growth factor, growth factor, and insulin-like growth factor may be secreted by neurotrophic mediators, which can promote nerve regeneration, tissue metabolism, and functional recovery to provide the needed richer environment $(18,19)$. Wogonin also plays a neuroprotective role through other special mechanisms, including anti-phagocytosis, antiimmunity, and antibacterial processes, along with improving lipid metabolism.

Taken together, wogonin could significantly improve the neurological function of rats with cerebral ischemia injury. The possible mechanism was that wogonin might promote the proliferation of cells in the ischemic region, increase the number of new blood vessels, reduce the apoptosis of neurons and inflammation edema in the ischemic brain tissue, and thus exert a protective effect for nerves. This protective effect may be related to TGF- $\beta 1$.

However, there are still many shortcomings in this study. There was no quantitative detection of cell death and inflammatory edema cell, and it should be noted that some studies have shown that wogonin has no or only minor neuroprotective effects on mature neurons $(7,9)$. Furthermore, higher concentrations may have toxic effects $(26,27)$. Despite this, some studies have found that exceeding $10 \mathrm{pM}$ did not reduce the oxidative stressinduced neuronal damage, although cytotoxic effects were not observed (7). It is still necessary to further study the effects of wogonin on ischemic cerebrovascular disease.

\section{Acknowledgments}

We would like to thank Qun Wang (Beijing Tiantan Hospital, Capital Medical University, Beijing, China) for editing the manuscript for English grammar.

Funding: This work was supported by The National Natural Science Foundation grant to Dr. Zhaohui Zhang (number: 81671051).

\section{Footnote}

Conflicts of Interest: The authors have no conflicts of interest to declare.

Ethical Statement: The authors are accountable for all aspects of the work in ensuring that questions related to the accuracy or integrity of any part of the work are appropriately investigated and resolved. All the experimental procedures follow the guidance of the Ministry of Science and Technology on experimental animals.

\section{References}

1. Hong KS, Saver JL. Quantifying the value of stroke disability outcomes: WHO global burden of disease project disability weights for each level of the modified Rankin Scale. Stroke 2009;40:3828-33.

2. Gong X, Sucher NJ. Stroke therapy in traditional Chinese medicine (TCM): prospects for drug discovery and development. Trends Pharmacol Sci 1999;20:191-6.

3. Shen SC, Lee WR, Lin HY, et al. In vitro and in vivo inhibitory activities of rutin, wogonin, and quercetin on lipopolysaccharide-induced nitric oxide and prostaglandin E2 production. Eur J Pharmacol 2002;446:187-94.

4. Hui KM, Huen MS, Wang HY, et al. Anxiolytic effect of wogonin, a benzodiazepine receptor ligand isolated from Scutellaria baicalensis. Biochem. Pharmacol 2002;64:1415-24.

5. Wakabayashi I, Yasui K. Wogonin inhibits inducible prostaglandin E (2) production in macrophages. Eur J Pharmacol 2000;406:477-81.

6. Shieh DE, Liu LT, Lin CC. Antioxidant and free radical scavenging effects of baicalein, baicalin and wogonin. Anticancer Res 2000;20:2861-5.

7. Chun W, Lee H, Kong P, et al. Synthetic wogonin 


\section{Page 10 of 10}

derivatives suppress lipopolysaccharide-induced nitric oxide production and hydrogen peroxide-induced cytotoxicity. Arch Pharm Res 2005;28:216-9.

8. Cho J, Lee HK. Wogonin inhibits excitotoxic and oxidative neuronal damage in primary cultured rat cortical cells. Eur J Pharmacol 2004;485:105-10.

9. Kang SS, Lee JY, Choi YK, et al. Neuroprotective effects of flavones on hydrogen peroxideinduced apoptosis in SH-SY5Y neuroblostoma cells. Bioorg Med Chem Lett 2004;14:2261-4.

10. Fan Y, Ding S, Sun Y, et al. MiR-377 Regulates Inflammation and Angiogenesis in Rats After Cerebral Ischemic Injury. J Cell Biochem 2018;119:327-37.

11. Longa EZ, Weinstein PR, Carlson S, et al. Reversible middle cerebral artery occlusion without cranietomy in rats. Stroke 1989;20:84-91.

12. Kong Z, Hong Y, Zhu J, et al. Endothelial progenitor cells improve functional recovery in focal cerebral ischemia of rat by promoting angiogenesis via VEGF. J Clin Neurosci 2018;55:116-21.

13. Brambrink AM, Koerner IP, Dichl K, et a1. The antibiotic Erythromycin induces tolerce against transient globaloerebral ischemia in rats. Anesthesiology 2006;104:1208-15.

14. Lee H, Kim YO, Kim H, et al. Flavonoid wogonin from medicinal herb is neuroprotective by inhibiting inflammatory activation of microglia, FASEB J 2003;17:1943-4.

15. Piao HZ, Jin H, Chun J, et al. Neuroprotective effect of wogonin: potential roles of inflammatory cytokines, Arch Pharm Res 2004;27:930-6.

16. Heo H, Shin Y, Cho W, et al. Memory improvement in ibotenic acid induced model rats by extracts of Scutellaria baicalensis. J Ethnopharmacol 2009;122:20-7.

17. Piao HZ, Jin SA, Chun HS, et al. Neuroprotective Effect of Wogonin: Potential Roles of Inflammatory Cytokines.

Cite this article as: Kong Z, Shen Q, Jiang J, Deng M, Zhang $Z$, Wang G. Wogonin improves functional neuroprotection for acute cerebral ischemia in rats by promoting angiogenesis via TGF- $\beta 1$. Ann Transl Med 2019;7(22):639. doi: 10.21037/ atm.2019.10.70
Kong et al. Wogonin improves neuro-function via TGF- $\beta 1$ in stroke

Arch Pharm Res 2004,27:930-6.

18. Son D, Leeb P, Leeb J, et al. Neuroprotective effect of wogonin in hippocampal slice culture exposed to oxygen and glucose deprivation. Eur J Pharmacol 2004;493:99-102.

19. Paczkowska E, Larysz B, Rzeuski R, et a1. Human hematopoietic stem progenitor-enriched CD34(+)cells are mobilized into peripheral blood during stress related to ischemic stroke or acute myocardial infarction. Eur J Haematol 2005;75:461-7.

20. Popa-Wagner A, Dinca I, Yalikun S, et al. Accelerated delimitation of the infarct zone by capillary-derived nestinpositive cells in aged rats. Curr Neurovasc Res 2006;3:3-13.

21. Zhao L, Chen Z, Zhao Q, et al. Developmental toxicity and genotoxicity studies of wogonin. Regul Toxicol Pharmacol 2011;60:212-7.

22. Qi Q, Peng J, Liu W, et al. Toxicological studies of wogonin in experimental animals. Phytother Res 2009;23:417-22.

23. Wang L, Zhang H, Chen B, et al. Effect of magnetic nanoparticles on apoptosis and cell cycle induced by wogonin in Raji cells. Int J Nanomedicine 2012;7:789-98.

24. Wang W, Guo QL, You QD, et al. The anticancer activities of wogonin in murine sarcoma $\mathrm{S} 180$ both in vitro and in vivo. Biol Pharm Bull 2006,29:1132-7.

25. Wang W, Guo Q, You Q, et al. Involvement of bax/bcl2 in wogonin-induced apoptosis of human hepatoma cell line SMMC-7721. Anticancer Drugs 2006;17:797-805.

26. Gao Z, Huang K, Yang X, et al. Free radical scavenging and antioxidant activities of flavonoids extracted from the radix of Scutellaria baicalensis Georgi. Biochim Biophys Acta 1999;1472:643-50.

27. Lee HH, Yang L, Wang C, et al. Differential effects of natural polyphenols on neuronal survival in primary cultured central neurons against glutamate- and glucose deprivation-induced neuronal death. Brain Res 2003;986:103-13. 